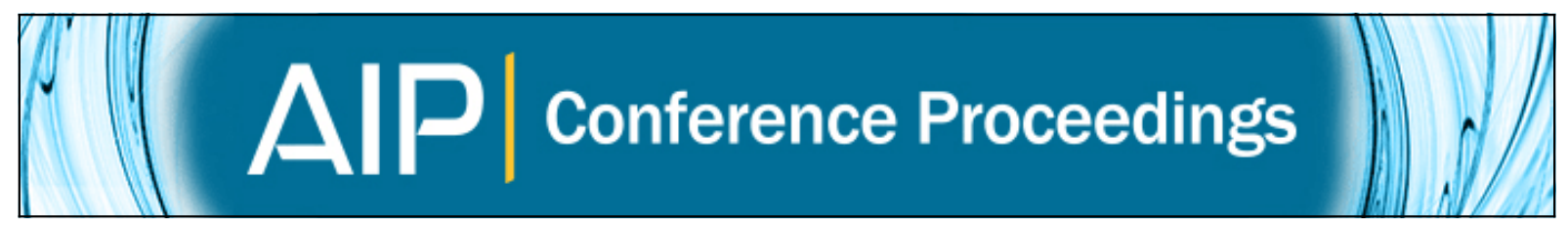

\title{
Heat Capacity of Neutron Transmutation Doped Ge Type 18
}

W. Holmes, J. J. Bock, and A. E. Lange

Citation: AIP Conference Proceedings 1185, 103 (2009); doi: 10.1063/1.3292293

View online: http://dx.doi.org/10.1063/1.3292293

View Table of Contents: http://scitation.aip.org/content/aip/proceeding/aipcp/1185?ver=pdfcov

Published by the AIP Publishing

\section{Articles you may be interested in}

Non-Debye heat capacity formula refined and applied to GaP, GaAs, GaSb, InP, InAs, and InSb

AlP Advances 3, 082108 (2013); 10.1063/1.4818273

SiGe Integrated Circuit/SQUID Hybrid Cryogenic Multiplexer for Superconducting Bolometer Array

AIP Conf. Proc. 1185, 534 (2009); 10.1063/1.3292398

On the heat capacities of Ta $2 \mathrm{Al} \mathrm{C}, \mathrm{Ti} 2 \mathrm{~S} \mathrm{C}$, and $\mathrm{Cr} 2 \mathrm{Ge} \mathrm{C}$

J. Appl. Phys. 104, 023526 (2008); 10.1063/1.2956511

Low-temperature contributions to the specific heat of GexAs40-xS60 glasses

AIP Conf. Proc. 899, 589 (2007); 10.1063/1.2733330

Carrier-type reversal in $\mathrm{Pb}-\mathrm{Ge}-\mathrm{Se}$ glasses: Photopyroelectric measurements of thermal conductivity and heat capacity

Appl. Phys. Lett. 78, 745 (2001); 10.1063/1.1345839 


\title{
Heat Capacity of Neutron Transmutation Doped Ge Type 18
}

\author{
W. Holmes*, J.J. Bock ${ }^{*, \dagger}$ and A.E. Lange** \\ * Jet Propulsion Laboratory, California Institute of Technology, 4800 Oak Grove Dr, Pasadena, CA, 91109 \\ ${ }^{\dagger}$ Department of Physics, California Institute of Technology, Mail Stop 59-33, Pasadena, CA, 91125 \\ ${ }^{* *}$ Department of Physics, Math and Astronomy, California Institute of Technology, Mail Stop 59-33, Pasadena, \\ CA, 91125
}

\begin{abstract}
We present measurements of the heat capacity of neutron transmutation doped (NTD) Ge temperature sensors from $100-300 \mathrm{mK}$. The NTD Ge sensor studied consists of a $30 \mu \mathrm{m} \times 100 \mu \mathrm{m} \times 250 \mu \mathrm{m}$ block of NTD Ge type 18 with the natural isotopic abundance, a doping of $n=5.6 \times 10^{16} \mathrm{~cm}^{-3}$ and ion implanted and metallized contact pads. Each sensor was mounted on a freestanding silicon nitride ( $\mathrm{Si}-\mathrm{N}$ ) pad supported by Si-N legs each with a cross section in the range 5-10 $\mu m^{2}$. Two of the Si-N legs were metallized for electrical readout of the NTD Ge sensor. The measured heat capacity of the NTD Ge sensor, which includes the metalization and Si-N pad, when fit to power law $C=C_{o} T^{\gamma}$ yields $C_{o}=4.3 \mathrm{pJ} / \mathrm{K}^{\gamma}$ and $\gamma=1.6$. The thermal conductance, $G_{S i-N}$, of the Si-N support legs was measured over a larger temperature range $80-800 \mathrm{mK}$. We find $G_{S i-N}$ at temperatures $<200 \mathrm{mK}$ of all 4 samples is at or below the $1 \mathrm{D}$ or quantum of thermal conductance limit.
\end{abstract}

Keywords: bolometers; heat capacity; NTD Germanium; cryogenics PACS: $65.60 .+\mathrm{a}$

\section{INTRODUCTION}

Bolometers currently fielded in telescopes to map the universe at millimeter and sub-millimeter wavelengths for astronomy and astrophysics $[1,2,3,4,5,6]$ use semiconducting neutron transmutation doped (NTD) Ge as the temperature sensor. [7] The response time of the bolometer, $\tau \sim C / G$, affects mainly the speed at which the pixel is chopped between two sources or the speed at which it scans over objects of interest. Here, $C$ is the heat capacity of the mm or sub-mm absorber and NTD Ge chip and $G$ is the thermal conductance of thermally isolating supports for the absorber and NTD Ge.[8] It is desirable to make $\tau$ as small as possible to reduce noise and systematic effects.

The NTD Ge chip is usually the largest contributor to the total heat capacity. Thus, it is important to know the heat capacity of the NTD Ge to properly design bolometers for real applications. Here, we present measurements of the heat capacity of a device, shown in the inset in Figure 1, consisting of $30 \mu \mathrm{m} \times 100 \mu \mathrm{m} \times 250 \mu \mathrm{m}$ NTD Ge type 18 indium bump bonded to a free standing silicon nitride (Si-N) island complete with metallized contact and two readout leads, all of which are suspended from a thick silicon frame by thin Si-N legs. This device is identical to the mounting of the NTD Ge on bolometers except it does not include a mm-wave or sub-mm wave absorbing mesh.

\section{SAMPLE PREPARATION AND TESTING}

The test samples were fabricated at JPL Microdevices Laboratory (MDL). [9] The processing starts with commercial "SOI" silicon wafers [10] 6 inches in diameter which have a $\sim 1 \mu \mathrm{m}$ thick $\mathrm{SiO}_{2}$ layer buried beneath $\mathrm{a} \sim 1 \mu \mathrm{m}$ thick, Si top layer on one side of the wafer. On each wafer, a Si-N film, $\sim 1 \mu \mathrm{m}$ thick, was grown on both sides of the wafer. Gold with a titanium adhesion layer was deposited and patterned on the side of the wafer with the buried oxide to define the electrical leads, and contact pads for wire bonding on the Si die. A nickel layer was deposited and patterned on the gold contacts prior to deposition of indium bump bonds. Once the metalization was complete, the Si-N film was patterned by plasma etching to define the contact pad island, thin suspension legs and the etch windows on the backside of the wafer. The samples were then diced in separate die and handled individually. The NTD Ge type 18 chips were then manually placed onto the contact pads on the island. Special tooling was used to press on NTD Ge with a controlled force to uniformly squash the indium bumps on to the contact pads. Then, the die were etched from the backside to remove the silicon to define the free standing structure. Four samples were prepared. Sample A consisted of the suspended NTD Ge with nominal contact metallization. Sample B was like sample A except with $6 \times$ the nominal volume of indium. Samples $\mathrm{C}$ and $\mathrm{D}$ were like sample $\mathrm{A}$ except with $6 \times$ the nominal volume of nickel.

For testing, each sample die was bonded into a gold plated copper carrier. Electrical contact to the sample 
die was made by wires wire-bonded from pads on the die to pads on a PCB board, complete with RF filtering and larger connector leads, in the carrier. Several carriers were mounted on a sample stage attached to the mixing chamber in an Oxford Kelvinox 50 dilution refrigerator for testing. The absolute temperature of the stage was measured using a calibrated germanium resistance thermometer (GRT) purchased from Lakeshore Cryotronics and recorded approximately once per second using an AVS 47 resistance bridge. The temperature of the sample stage was controlled with heaters on the samples stage and a TS530 PID controller coupled with the analog signal from resistance bridge monitoring the GRT. The precision of the control was typically better than $10 \mu \mathrm{K}$ peak to peak. The dilution refrigerator insert was equipped with low noise readout amplifiers adapted from bolometric array receivers. [1, 11]

The samples were biased by applying voltage to cold load resistors $R_{l}=20 M \Omega$ mounted on the mixing chamber of the dilution fridge to produce a DC bias current $I_{b}$. The sample voltage was amplified at two DC couple stages, the first was a JFET follower stage, with unity gain, mounted in the cryogenic space and the second was a differential amplifier, with a gain of 100, mounted over the warm feedthrough on the dilution insert top flange. Additional RF filters were used for all bolometer bias and readout wires routed from the cold JFET module to the second stage amplifier. Voltages for all measurements were digitized using a National Instruments 6031E data acquisition card. All test sequences were automated using Labview software.

\section{MEASUREMENT TECHNIQUE}

Determining $C(T)$ of each sample requires three measurements, $G(T), R(T)$ and $\tau$, and a model for the bolometric response. $[8,12,13,14]$ The values of $G(T)$ and $R(T)$ were determined from bolometer load curves $V_{o}\left(I_{b}\right)$ at sample stage temperatures $0.08 K<T_{o}<5 K$, where $V_{o}$ is the voltage across the bolometer. For each load curve, we stepped through a series of positive and negative $I_{b}$ centered about $I_{b}=0$. The time between each new bias point was $>1 \mathrm{sec}$ to ensure that the sample response time did not effect the measurements. We fit a line to the voltage at the output of the amplifier at low bias through $I_{b}=0$. The slope was used to determine $R$ at each sample stage temperature $T$. The $R(T)$ at $I_{b}=0$ was fit to the function $R(T)=R_{\text {leads }}+R_{o} \exp \left(\sqrt{T_{g} / T}\right)$, for variable range hopping (VRH) conduction characteristic of this NTD Ge type [7], where $R_{\text {leads }}$ is the lead resistance.

For our NTD Ge type 18 samples, the typical values of the fit parameters are $R_{o} \sim 80 \Omega$ and $T_{g} \sim 17.5 \mathrm{~K}$. The fits were good over the entire temperature range indicat- ing that stray RF power or infrared power in our system is negligible. The output voltage offset determined from the fit near $I_{b}=0$ was subtracted from the signal voltage to determine $V_{o}$. Using the VRH fit parameters and value of $R\left(I_{b}\right)=V_{o}\left(I_{b}\right) / I_{b}$, we determined $T$ at each point in the load curve. Then, we fit the bias power $\left(P_{b}=V_{o} I_{b}\right)$ as a function of bolometer temperature to the form $P_{b}=\left[g_{o} /(\beta+1)\right]\left(T^{\beta+1}-T_{o}^{\beta+1}\right)$ to determine the thermal conductance, $G(T)=g_{o} T^{\beta}$. From the quality of the fit, we find that non-thermal non-ohmic effects $[15,16]$ for these samples are negligible. We subtracted the contribution of both $3 \mathrm{k} \Omega$ leads, using the WiedmannFranz law [17], from the raw measured $G(T)$. The resulting $G(T) / N_{\text {leg }} T$ is shown in Figure 1. Here, we divide the number of Si-N legs on each sample to show the average conductance per Si-N leg.

The response time was measured by the response of the bolometer to a step in bias current. The bolometric model response of the samples to a step in $I_{b}$ is described by the equivalent circuit shown in Mather [14] with a capacitor in parallel with the bolometer. The response time of our bias circuit in a channel where the bolometer is replaced by a fixed resistor is $\tau_{e}=C_{S} /\left(1 / R+1 / R_{l}\right)$.

The response of the resistor and the samples were measured simultaneously using a bias step $\pm 5 \%$ of the mean $I_{b}$. The response time of the resistor, $R=10.1 \mathrm{M} \Omega$ was $\tau_{e}=0.82 \pm 0.4 m s$. At the highest bolometric resistance in our measurements, $R=51 M \Omega$, the electronic response time, $\tau_{e} \sim 1.2 \mathrm{~ms}$, is much faster than the fastest response time for all samples $\tau \sim 10 \mathrm{~ms}$. When $\tau_{e}$ is much faster than the thermal response time $\tau$, the transient response of the bolometer voltage in this equivalent circuit is a single exponential decay $\delta V_{o} \propto e^{-t / \tau}$ where

$$
C(T)=\tau\left(G(T)-\alpha P_{b}+2 \alpha P_{b} R /\left(R+R_{l}\right)\right) .
$$

Phase errors at the $2.5 \mathrm{kHz}$ sample rate were negligible as determined by the averaging of the resistor response. The step period, $3.27 \mathrm{sec}$, was adjusted so that the transient response of the slowest sample had decayed by a factor $>1000$ before the next bias step was applied.

The resistor and samples B, C and D, display simple single pole exponential decay. Sample A, however, has a two component response at all temperatures $100-$ $300 \mathrm{mK}$. The fast component, between $10<\tau<25 \mathrm{~ms}$, follows a single exponential decay. Due to the dependence on temperature and bias, we associate the fast component with the thermal response of the NTD Ge on the island. The heat capacity for sample A, deduced from the fast component using Equation 1, is shown in Figure 2 . The second component was only about $1 \%$ of the total response. The $3 d B$ decay time of the slow component, computed in a region where the fast component is not significant, is $\sim 50 \mathrm{~ms}$. This slow component is present in the data over the entire temperature range and does not 


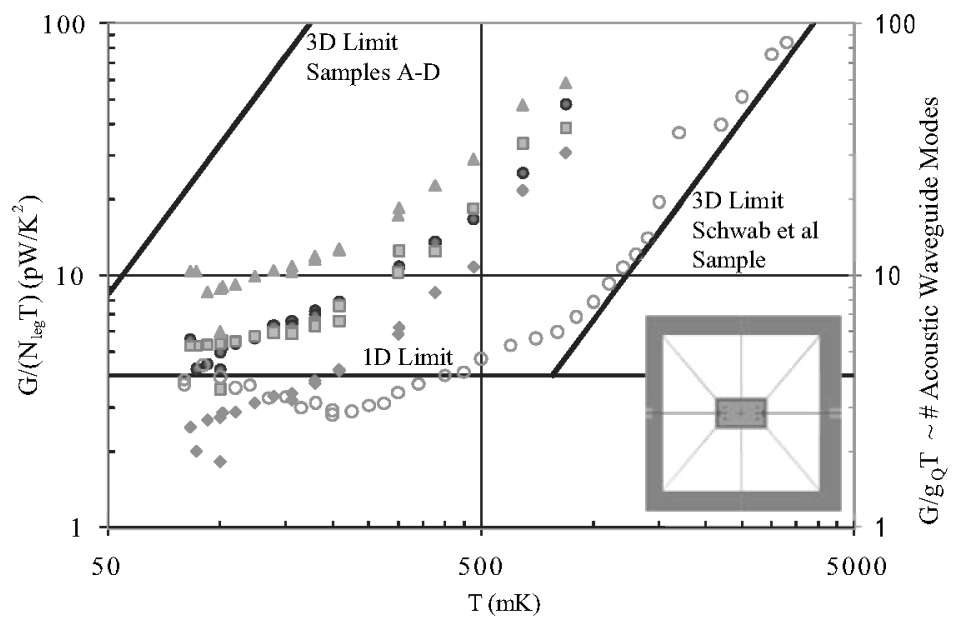

FIGURE 1. Measured $G(T) / T$ per Si-N leg (left y axis) as a function of temperature for sample A (solid dot), B (square), $\mathrm{C}$ (diamond) and D (triangle). The data for the sample measured by Schwab et al are plotted as open dots.[18] The G(T) data, normalized by the quantum of thermal conductance $g_{O} T$ (right y-axis), yields the approximate number of acoustic waveguide modes conducting heat through the Si-N leg. The 3D limit for our samples and the Schwab sample, both of which are computed without adjustable parameters, and the 1D limit, which is the same for all samples, are plotted as solid lines. Inset on bottom right: NTD Ge mounted on a Si-N pad and suspended by $\sim 1 \mu m$ thick Si-N legs. The two widest legs (10 $\mu m$ each), running horizontally in the figure, contain thin gold metallization to make electrical contact to the NTD Ge. The other 6 legs are unmetallized and $5 \mu m$ wide each.

depend on bias. A similar behavior has been measured in Si-N bolometers. $[3,5]$ Conclusive identification of this component would require more measurements. The most likely physical mechanism for this response is a slow mechanical relaxation process at dislocations in the lattice of the amorphous Si-N. [19]

\section{DISCUSSION}

An alternate way to represent the thermal conductance in electrical insulators at low temperatures, such as Si-N, is to consider propogation of power by acoustic modes through the leg. This is analogous to electromagnetic power transmitted through a waveguide, but different in one signficant way. In an acoustical system, there are 4 "massless" modes that do not have a low frequency cutoff and thus propagate at $T=0$. [20] By dividing the $G(T)$ data by the quantum of thermal conductance $g_{Q} T=T k_{b}^{2} \pi^{2} / 3 h=0.946 T \mathrm{pW} / \mathrm{K}$, right $\mathrm{y}$-axis in Figure 1 , the $\mathrm{G}(\mathrm{T})$ measured is reduced to an approximate number of acoustic modes carrying the heat from one side of the leg to the other. The $1 \mathrm{D}$ limit for thermal conduction is defined when the temperature is low enough that only these 4 modes can propogate heat. [20] Thermal conductance in this limit has been measured [18], as shown in Figure 1, for samples at temperatures where the leg dimensions $85 \mathrm{~nm}-200 \mathrm{~nm}$ are less than the dominant phonon wavelength $\lambda_{d} \sim 75 \mathrm{~nm} / T$.
Since our samples are much thicker, $1 \mu \mathrm{m}$, we expect the transition to the 1D limit to be at $T<75 \mathrm{mK}$ but sample $\mathrm{C}$ is at the $1 \mathrm{D}$ limit at $T \sim 200 \mathrm{mK}$. To understand what is going on in our samples, we consider the limit of maximum or upper limit to the conductance. This occurs when there is no bulk scattering of phonons and only specular scattering of the phonons off of the leg surfaces. The computed $3 \mathrm{D}$ upper limit ${ }^{1}$, which does not have any adjustable parameters, for the sample measured by Schwab et al [18] lies nearly on the measured data for $T>1 K$ to the temperature of the cross over to the $1 \mathrm{D}$ limit, as shown in Figure 1. Thus, bulk and non-specular surface scattering are negligible over the $\sim 3 \mu \mathrm{m}$ length of the legs in the Schwab sample. In our samples, the 3D upper limit, $G=157 A T^{3} \mathrm{pW} / \mathrm{K}$, is higher than the measured $G(T)$ at all temperatures. Here $A=5 \mu m^{2}$ is the cross sectional area of the samllest $\mathrm{Si}-\mathrm{N}$ leg. Thus, as indicated on the right y-axis in Figure 1, dozens of higher order acoustical modes could, but do not contribute to

\footnotetext{
1 The support legs in the sample tested by Schwab, et. al., consisted of $t_{S i-N}=60 \mathrm{~nm}$ thick Si-N and $t_{N b}=25 \mathrm{~nm}$ thick Nb leads. Since the lowest mode phonons have a slowly varying (or constant) strain across the cross section, we computed the weighted average of the mechanical properties, for example $c_{11}^{e f f}=\left(t_{N b} C_{11}^{N b}+t_{S i} C_{11}^{S i-N}\right) /\left(t_{N b}+t_{S i-N}\right)$. We use these effective materials parameters then to compute the sound velocities, $v_{i}$, needed in the expression for the "phonon" Stefan-Boltzman constant $\sigma=\Sigma_{i} \pi^{5} k_{b}^{4} / 15 h^{3} v_{i}^{2}$ that defines the 3D upper limit for heat flow in any material.[21]
} 


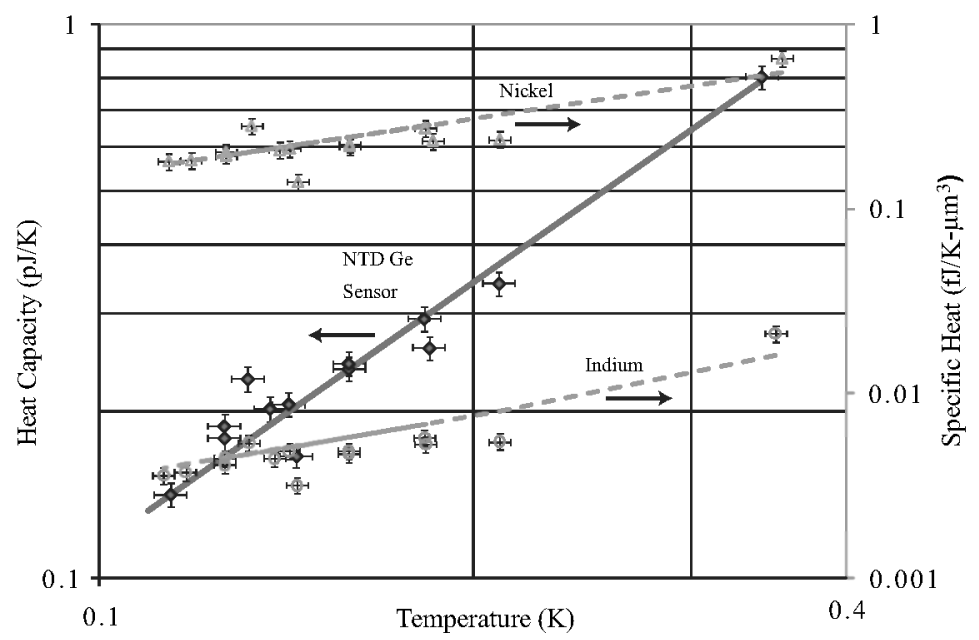

FIGURE 2. Heat capacity (left y-axis) for the NTD Ge 18 mounted on the contact pad island and specific heat (right y-axis) for the indium and nickel contact layers as a function of temperature. A power law (solid line) $C=C_{0} T^{\gamma}$ is fit to the NTD Ge sensor data. The function $a T+b T^{3}$ (dotted line) is fit to the specific heat data, where the $a$ is variable and $b$ is fixed at the computed Debye specific heat.[17]

thermal conduction at all temperatures of our measurement. Thus, bulk and/or non-specular surface scattering of these high order acoustical modes must be significant over the length of legs, $>500 \mu \mathrm{m}$, of our samples.

The measured heat capacity data for the NTD Ge 18 sensor is shown in Figure 2. The contributions from each component are shown in Table 1. To determine the specific heat of the extra indium on sample B, and the extra nickel on samples $C$ and $D$, we subtracted the measured value of heat capacity for sample $\mathrm{A}$ from each. For the indium bump bonds, we measure an electronic component to the specific heat $0.037 T$ which is $\sim 1 / 3$ of the value expected [17] if the entire volume were in the normal state. This suggests that the superconducting transition temperature of the squashed indium bumps has been depressed below the bulk value $T_{c}=3.4 \mathrm{~K}$.

We measure the specific heat of the nickel contact layer to be $\sim 1.54 \mathrm{TfJ} / \mathrm{K}^{2} \mathrm{\mu m}^{3}$ which is $50 \%$ larger than the value for bulk. [17] We estimate our experimental error, dominated by the knowledge of the nickel layer volume, to be $10 \%$. This excess specific heat for the nickel could be due to alloying of the metals in the contacts. As listed in Table 1, the calculated heat capacity of just the contact metals accounts for all of the measured heat capacity. Given our experimental error, the heat capacity of the NTD Ge material is in the range $10-$ $20 \mathrm{fJ} / \mathrm{K}$ at $120 \mathrm{mK}$ or about $4 \times$ the specific heat expected for the lattice. The measured value of heat capacity of NTD Ge type 34B, scaled proportional to doping density, $[22,23]$ would give $C=0.0006 T \mathrm{fJ} / \mathrm{K}-\mu \mathrm{m}^{3}$ for NTD
TABLE 1. Heat capacity of components of the NTD Ge type 18 sensor.

\begin{tabular}{lrrr}
\hline & $\begin{array}{r}\text { Volume } \\
\mu \mathbf{m}^{\mathbf{3}}\end{array}$ & $\begin{array}{r}\text { Specific Heat } \\
\left(\mathbf{f J} / \mathbf{K}^{\mathbf{2}}-\mu \mathbf{m}^{\mathbf{3}}\right)\end{array}$ & $\begin{array}{r}\text { Heat Capacity } \\
\mathbf{f J} / \mathbf{K} \text { at 120 } \mathbf{m K}\end{array}$ \\
\hline $\mathrm{Ge}$ & 875,000 & $<0.0006 T$ & $<67$ \\
$\mathrm{In}$ & 17,000 & $0.037 T$ & 78 \\
$\mathrm{Au}$ & 940 & $0.071 T$ & 8 \\
$\mathrm{Ni}$ & 380 & $1.5 T$ & 70 \\
$\mathrm{Ti}$ & 76 & $0.032 T$ & 3 \\
$\mathrm{Si}-\mathrm{N}$ & 93,200 & $0.002-0.006$ & $200-600$ \\
Sample A measured & & $152 \pm 15$ \\
\multicolumn{2}{l}{ Contact metals only heat capacity } & $159 \pm 16$ \\
\hline
\end{tabular}

Ge type $18 .^{2}$ Thus, our measurements indicate the heat capacity of the NTD Ge 18 is $3-4 \times$ lower than expected by simple scaling of measurements on NTD type 34B. Finally, the second largest contributor to the total volume on the suspended island after the NTD Ge, is the Si-N pad.

In Table 1, we compute the value of the heat capacity of the Si-N as suggested by two sets of published measurements $[6,19]$ both of which are more than our measured heat capacity. For sample A, the upper bound on the specific heat for the Si-N is very small $<0.0001 \mathrm{fJ} / \mathrm{K}_{\mu} \mathrm{m}^{3}$.

In conclusion, we have measured the specific heat

\footnotetext{
${ }^{2}$ If the electrons in the NTD Ge were treated as a free electron gas, the Fermi temperature defined in the usual way is $T_{f} \propto N^{2 / 3} \sim 6.2 \mathrm{~K}$ and the specific heat is $C=0.0006 T \mathrm{fJ} / \mathrm{K}-\mu \mathrm{m}^{3}$.
} 
of an NTD Ge type 18 sensor with contact metallizations on a free standing $\mathrm{Si}-\mathrm{N}$ island and suspended by thin Si-N legs. The heat capacity data were fit to a power law $C(T)=4.3 T^{1.6}$ over the temperature range $100-300 \mathrm{mK}$. The heat capacity of the block of NTD Ge at these temperatures is dominated by the contact metalizations and indium bump bonds. The specific heat of the Si-N island is significantly smaller than values reported for other samples. This indicates that excess heat capacity is not a generic feature of Si-N and is strongly sample dependent. The measured thermal conductance of $>500 \mu \mathrm{m}$ long Si-N legs with $5-10 \mu \mathrm{m}^{2}$ cross sectional area that support the NTD Ge are near the 1D limit of thermal conductance at temperature $<200 \mathrm{mK}$. At these temperatures, dozens of high order acoustical modes should contribute to the thermal conductance of our samples but do not in our samples. By comparison with measurements of the thermal conductance on much smaller samples, we estimate the mean free path for phonons in these high order modes is in the range $10-100 \mu \mathrm{m}$.

\section{ACKNOWLEDGMENTS}

We acknowledge the help of Mihhee Yun for fabricating the samples and George Sirbi for mounting them in the copper carrier. This research was carried out at the Jet Propulsion Laboratory, California Institute of Technology, under a contract with the National Aeronautics and Space Administration.

\section{REFERENCES}

1. B. P. Crill, et al., ApJS 148, 527-541 (2003).

2. J. Hinderks, et a1., $A p J$ 692, 1221-1246 (2009).

3. Y. D. Takahasi, et al., "CMB polarimetry with BICEP: instrument characterization, calibration, and performance," in Proc. of SPIE, edited by J.C.Mather, 2008, vol. 7020, p. 70201D.

4. L. Earle, et al., "Z-Spec: a broadband direct-detection millimeter-wave spectrometer -instrument status and first results," in Proc. SPIE, 2006, vol. 6275, p. 6275E.

5. J.-M. Lamarre, et al., in preparation for Astronomy and Astrophysics (2009).

6. W. Holmes, J. Bock, B. Crill, W. Jones, T. C. Koch, A. Lange, and C. Paine, Appl. Opt. 47, 5996-6008 (2008).

7. E. E. Haller, IR Phys. 25, 257-266 (1985).

8. P. Richards, J. Appl. Phys 76, 1-24 (1994).

9. M. Yun, T. Koch, J. Bock, W. Holmes, L. Wild, J. Mulder, A. Turner, C. Paine, and A. Lange, "Bolometric Detectors for the Planck Surveyor," in Proc. of SPIE, edited by T. Phillips, and J. Zmuidzinas, 2003, vol. 4855, pp. $136-147$.

10. Analog Devices Belfast LTD., Hannahstown Hill, Belfast, Northern Ireland, UK, Buried silicon oxide wafers (1999).
11. C. Paine, J. Bock, V. Hristov, and A. Lange, "A Low Noise, High Thermal Stability, 0.1K Test Facility for the Planck HFI Bolometers," in Adv. Cryo. Eng., edited by L. Summers, Plenum Press, New York, 2001, vol. 47, pp. 1651-1658.

12. R. V. Sudiwala, M. Griffin, and A. Wooderaft, Int. J. of IR and $m m$-Waves 23 (2002).

13. M. Griffin, and W. Holland, Int. J. of IR and mm-Waves 9, 861-875 (1988).

14. J. C. Mather, Appl. Opt. 23, 584-588 (1984).

15. M. Galeazzi, and D. McCammon, J. Appl. Phys. 93, 4856 (2003).

16. M. Piat, J.-P. Torre, E. Breelle, A. Coulais, A. Woodcraft, W. Holmes, and R. Sudiwala, Nucl. Instrum. Methods A 559, 588-590 (2006).

17. C. Kittel, Introduction to Solid State Physics, John Wiley and Sons Inc., New York, 1986, 6th edn.

18. K. Schwab, E. Hendriksen, and M. Roukes, Nature 404, $974(2000)$.

19. M. Kenyon, P. Day, C. Bradford, J. Bock, and H. Leduc, J. Low Temp. Phys. 151, 112-118 (2008).

20. L. Rego, and G. Kirczenow, Phys. Rev. Lett. 81, 232 (1998).

21. T. Klitsner, and R. O. Pohl, Phys. Rev: B 36, 6551-6565 (1987).

22. E. Olivieri, M. Barucci, J. Beeman, L. Risegari, and G. Ventura, J. Low Temp. Phys. 143, 153-161 (2006).

23. M. Barucci, J. Beeman, E. Olivieri, E. Pasca, L. Risegari, and G. Ventura, Physica B 368, 139-142 (2005). 